\title{
INVARIANTS OF NUMBER FIELDS RELATED TO CENTRAL EMBEDDING PROBLEMS
}

\section{H. OPOLKA}

\author{
Mathematics Institute \\ Bunsenstrasse 3-5 \\ D-3400 Gott Ingen
}

(Received December 7, 1988)

\begin{abstract}
Every central embedding problem over a number field becomes solvable after enlarging its kernel in a certain way. We show that these enlargements can be arranged in a universal way.
\end{abstract}

KEY WORDS AND PHRASES. Central embedding problems, strict cohomological dimension, Leopoldt-conjecture.

1980 AMS CLASSIFICATION CODES. 12A55, 12A60

1. CENTRAL EMBEDding PROBLEMS.

Let $K$ be a number field and let $p$ be a prime number. Then there is a smallest natura1 number $t=t(k, p)$ depending on $1 y$ on $k$ and $p$, the so called p-exponent of $k$, with the following properties:

(1) Every central embedding problem $E_{m}=E\left(G, Z / p^{m}, c\right)$ for the absolute Galois group $G_{k}=G a l(\bar{k} / k)$ of $k$, where $G=G a l(K / k)$ is the Galols group of a finite Galols subextension $K / k$ of $\bar{k} / k$ which is ramified only at $p$ and $\infty$ and where $k(\mu) / p$ is cyclic, has exponent $<2 \mathrm{~m}+t$. Recall that $\mathrm{E}_{\mathrm{m}}$ is solvable, i.e. there is an epimorphism $\Psi: G_{k} \rightarrow G(c)$ of $G_{k}$ onto the central group extension $G(c)$ defined by the co-cycle $c: G \times G \rightarrow Z / p^{m}$ such that $\Psi$ composed with the natural map $G(c) \rightarrow G$ yields the given epimorphism $G_{k}+G$, if and only if the class of (c) becomes trivial in the Brauer group $\operatorname{Br}\left(\mathrm{k}_{\mathrm{p}}\left(\mu_{\mathrm{m}}\right)\right)$ of $\mathrm{k}_{\mathrm{p}}\left(\mu_{\mathrm{m}}\right), \mu_{\mathrm{p}} \mathrm{m}=$ group of roots of unity of $\mathrm{k}^{*}$ of order dividing $\mathrm{p}^{\mathrm{m}}$; this means that if $x_{\mathrm{m}}: \mathrm{z} / \mathrm{p}^{\mathrm{m}}+\mu_{\mathrm{p}}$ is an isomorphism then (c) becomes
trivial under the map

$$
\tilde{x}_{m}: H^{2}\left(G, Z / p^{m}\right) \stackrel{i n f}{\rightarrow} H^{2}\left(G_{k}, Z / p^{m}\right) \stackrel{r}{\rightarrow} H_{H^{2}}\left(G_{k}\left(\mu_{p}\right) z / p^{m}\right) \stackrel{x_{m}^{*}}{\rightarrow} H^{2}\left(G_{k}\left(\mu_{p}\right),,^{*}\right) \cong B r\left(k\left(\mu_{p}\right)\right)
$$

where $x_{m}^{*}$ is the map induced by $x_{m}$ on cohomology see Hoechsmann, [1]). The exponent of $E_{m}$ is the sma1lest natural number $n \geqslant m$ such that the embedding problem $E_{n}$ which is obtained from $E_{m}$ by considering the co-cycle $c: G \times G+Z / p^{m}+Z / p^{n}$ is solvable. 
In order to prove (1), choose for any natural number $\hat{\mathrm{m}}>\mathrm{m}$ an isomorphism

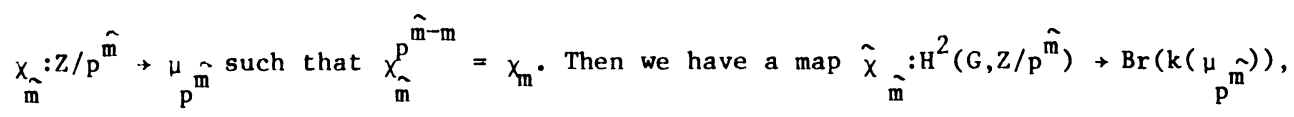
and the resulting diagram relating $\hat{x}_{m}$ and $\hat{x}_{\hat{m}}$ commutes. Since $\hat{x}_{\hat{m}}((c))$ can be represented by a Galols co-cycle all of whose values are roots of unity, the algebra class $\hat{\chi}_{\hat{m}}((c))$ splits and only if it splits locally at all places above $p$ and $\infty$ and this is the case if it splits at $\infty$ and

$$
\left(k_{v}\left(\mu_{p}\right): k_{v}\left(\mu_{p}\right)\right) \equiv 0 \bmod p^{m} \text { for al1 v above } p \text {; }
$$

(see classels [2], p. 191, $10.5 \mathrm{ff}$ ). It is clearly possible to find a smallest integer $d=d(k, p)$ depending only on $k$ and $p$ such that $\hat{x}_{\hat{m}}((c))$ splits with $\hat{m}=2 m+d$. For instance, for $k=Q$ we can take $d=d(Q, p)=0$ for all p. The $p$-exponent of $E_{m}$ is the smallest natural number $n>m$ such that the induced embedding problem $E_{n}$ has a solution which is ramified only at $p$ and $\infty$. The smallest integer $s>0$ such that the $p$-exponent of every $E_{m}$ is $<2 m+s$ is called the strong $p$-exponent of $k$ (if it exists).

(2) If $p$ does not divide the class number of $Q\left(\mu_{p}\right)$ then the strong p-exponent of every cyclotomic field $k=Q\left(\mu_{p}\right)$ exists and is equal to its (usual) p-exponent.

This can be shown as follows: Let $E_{m}$ be a central embedding problem for $G_{k}$. Then for $t=t(k, p)$ the induced embedding problem $E_{2 m}+t$ is solvable. The assumption implies that $p$ does not divide the class number of $Q\left(\mu_{p}\right)$ for every 1 (see Iwasawa [3]). Therefore the Galois theoretic obstruction to the existence of a solution which is unramified outside $p$ and $\infty$ as described in Neukirch [4], (8.1), is trivial.

The p-adic Leopoldt conjecture for $k$ implies that $H^{2}\left(G_{k}(p), Q / Z\right)=0$, where $G_{k}(p)$ is the Galois group of the maximal p-extension $k^{p} / k$ which is unramified outside $p$ and $\infty$. This shows that every central embedding problem $E_{m}$ for $G_{k}(p)$ has finite $p-$ exponent, (see Opolka [5], (5.2)). Does this imply that the strong p-exponent of $k$ is finite? If so, how is it related to the usual p-exponent of $k$ ? Conversely, if the strong $p$-exponent of $k$ is finite then $H^{2}\left(G_{k}(p), Q / Z\right)=0$ and the $p$-adic Leopoldt conjecture holds for $k$.

\section{REFERENCES}

1. HOECHSMANN, K., Zum Einbettungsproblem, JRAM, 229(1968), 81-106.

2. CASSEls, J.W.S., A. Frôhl1ch: Algebra1c Number Theory, Ac. Press, London, 1967.

3. IWASAWA, K., A Note on Class Numbers of Algebraic Number Fields, Abh. Math. Sem. Hamburg, 20 (1956), 257-258.

4. NEUKIRCH, J., Über das Einbettungsproblem der Algebraischen Zahlentheorie, Inventiones Math., 21 (1973), 59-116.

5. OPOLKA, H., Cyclotomic Splitting Fields and Strict Cohomological Dimension, Israe1 J. of Math., 52 (1985), 225-230. 


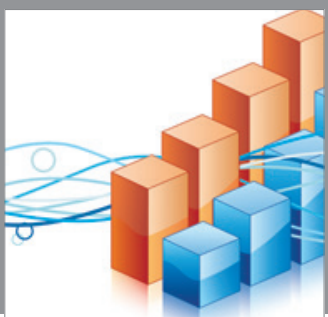

Advances in

Operations Research

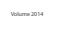

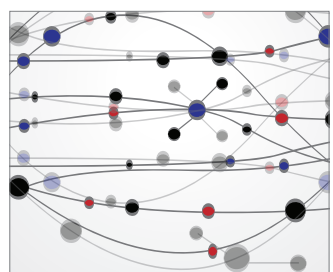

\section{The Scientific} World Journal
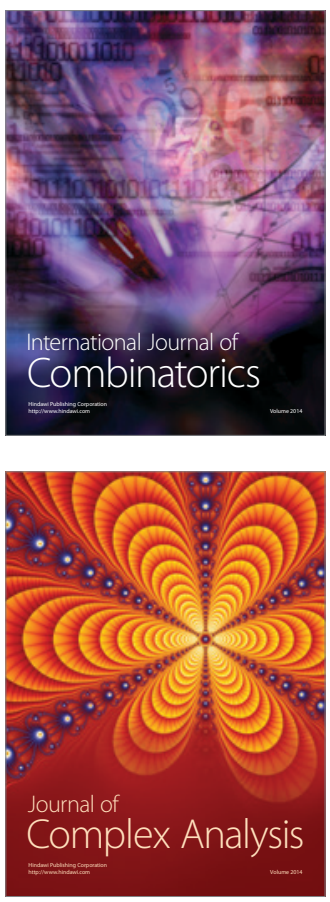

International Journal of

Mathematics and

Mathematical

Sciences
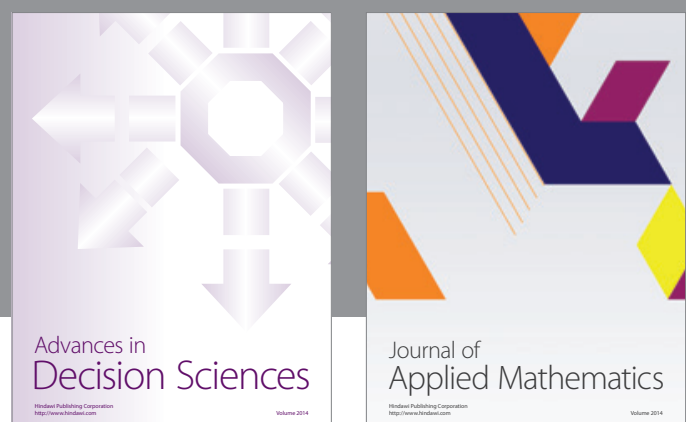

Journal of

Applied Mathematics
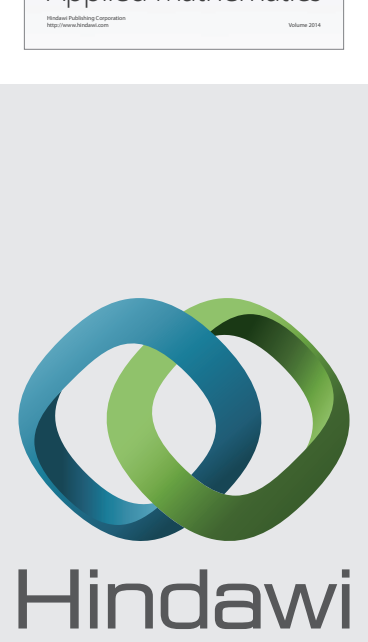

Submit your manuscripts at http://www.hindawi.com
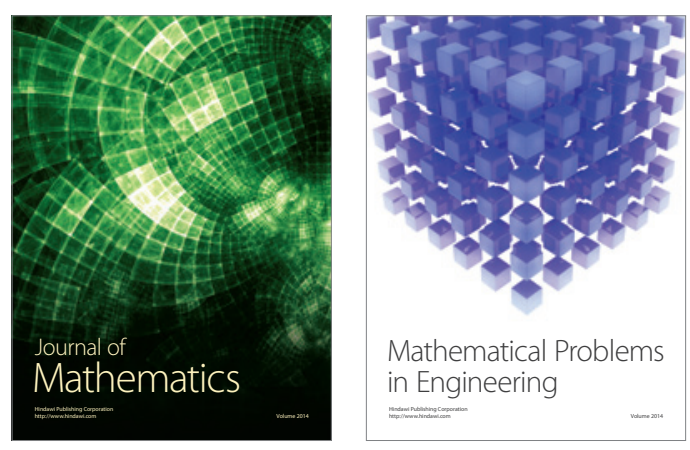

Mathematical Problems in Engineering
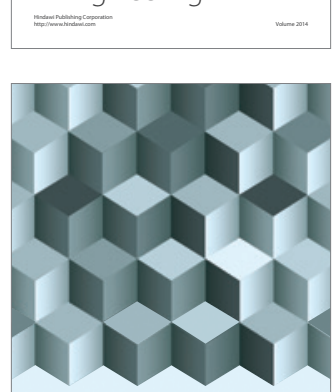

Journal of

Function Spaces
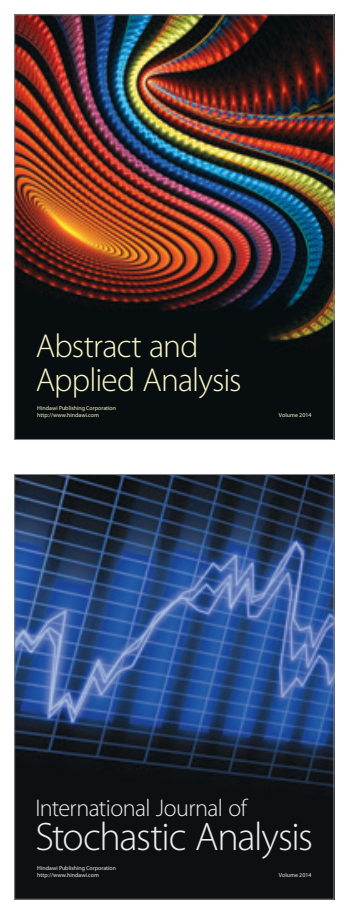

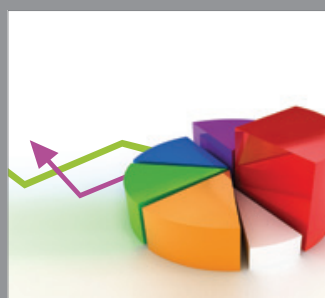

ournal of

Probability and Statistics

Promensencen
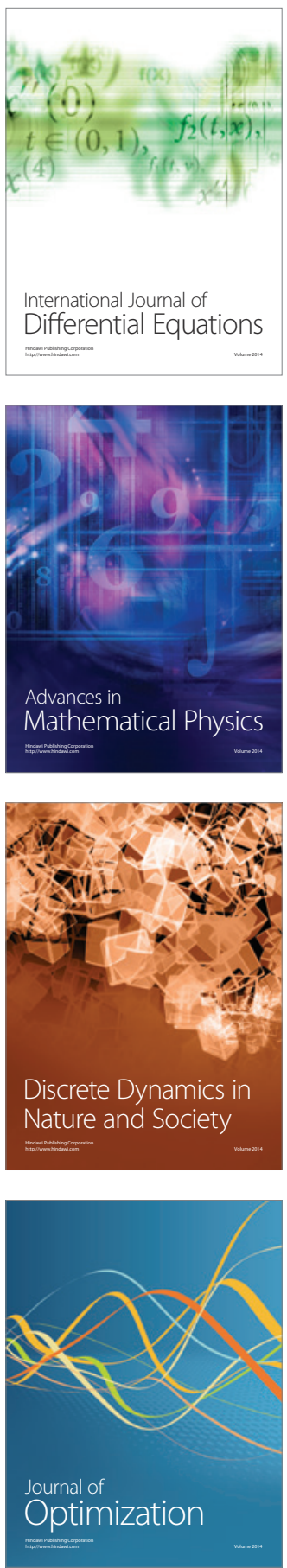